\section{DER INTERESSANTE FALL}

\author{
L.M., 74 Jahre alt, Konsultationen ab September 2020
}

Symptome: Blasenbeschwerden und Schlafstörungen

Wohnort: 6 Jahre alte Minergie-Attikawohnung

\section{Anamnese}

Urologische Befunde: September 2020: keine namhaften pathologischen Befunde furr die Symptome des stetigen Harndrangs. Die Patientin war in urolog. Spezialarztpraxis gründlichst abgeklärt. Eine antibiotische Therapie war erfolglos. Die Urologin befürwortete den Therapieversuch mit Akupunktur. So gelangte die Patientin zu mir. Ich kannte sie von früheren Behandlungen, hatte sie aber seit Juni 2016 nicht mehr gesehen.

\section{Befunde}

Hauptbeschwerden: stetiger Harndrang, 2- bis 4-mal Nykturie und unruhiger, nicht erholsamer Schlaf.

Nebenbefunde: qualitative Verschlechterung der Haarqualität.

\section{Befunde der Ohrakupunktur}

Patientin hat seit ca. 1 Jahr 4 neue Titanzahnimplantate (Gebisskontrolle mit Frequenz $363 \mathrm{~Hz}$ nach Bahr ohne pathologische Resonanzen, was eine dentogene Ursache unwahrscheinlich macht).

Aurikulo-Testung (Patientin ist Rechtshänderin): starker Störherd Bereich Lunge rechts/Vit. B6 gekoppelt. Der Störherd hat Resonanz auf Umwelttoxine: Schimmelpilz Aflatoxin/Potenz MMM10, andere Noxen wie Viren, Bakterien etc. sind ohne Resonanz.

Symptomrelevanter Punkt: GB 43 (Ursache: Leber-/ Gallefunktion) passend zur Toxinbelastung.

TUP (tiefer Ursachenpunkt n. Reininger $7232 \mathrm{~Hz}$ ) liegt bei Niere 7 in Go, Silber: Ma 40 nicht dominant (Schlacken).

\section{Möglicher Pathomechanismus}

Toxin überfordert die Leber, sie bräuchte eine Unterstützung mit einem Medikament zur Drainage des Toxins in die Gallenwege. Da dies bisher spontan und ohne entsprechende Medikation nicht geschieht, wird der Blutreinigungsmechanismus über die Nierenfunktion erledigt und liefert in den Harnwegen irritierende Urinkomponenten, die über die Ureterenausgänge am Blasenboden zu sehr unangenehm brennenden Schmerzreizen im Bereich des Blasenbodens führt. Erst die ausleitende, die Leberfunktion stützende Medikation von Desmodium Fa. Phytolis konnte die Urinsymptome reduzieren. Desmodium (unterstützt die Leberdrainage) ist eine aus Afrika und Südamerika stammende Pflanze, welche die Leberfunktionen bez. Entgiftung verbessert. Dieses Medikament wurde der Patientin in der Dosierung $2 \mathrm{Kps}$./Tag verordnet.

Auf der Suche nach der Quelle des Toxins reagierte von 11 Staubproben aus derWohnung nur diejenige des Schlafzimmers der Patientin mit einer positiven Resonanz auf Aflatoxin.

Wenige Tage später sendet mir die Patientin ein erstes Bild von der Zimmerdecke des Schlafzimmers, ein zart bräunlich gefärbter Fleck (Abb. 1 innerhalb der 3 Punkte). Was tun? Meine Testung auf dem PC-Bild ergibt einen positiven RAC mit Aflatoxin über dem Fleck.

Die Patientin meldet dies dem Hausbesitzer, welcher sehr kollaborativ Handwerker mobilisiert. Diese öffnen den Dachraum und Abb. 2 illustriert deutlich, was die fehlerhafte Spenglerarbeit beim Neubau 2013 durch die Wasserinfiltration für einen gefährlichen Schaden am Gebälk angerichtet hat. Laut Baufachleuten muss das ganze Dach neu rekonstruiert werden, dies wird auf Frühjahr 2021 geplant.

Das gesamte verschimmelte Isolationsmaterial musste ebenfalls entfernt werden (Abb. 3).

Die Schlafzimmerdecke wurde provisorisch verschlossen und die Staubproben bleiben ohne RAC aufAflatoxin. Kleider,Teppiche etc., welche positiv auf Aflatoxin resonant waren, wurden nach 1- bis 2-maliger hochqualitativer Reinigung Aflatoxin-frei.

Inzwischen ist die Aflatoxinbelastung unter ausleitenden Medikamenten bei der Patientin in hoher MMMM-Potenz und betrifft noch die Milz. Patientin ist zuversichtlich.

Ende 2020 kommt die Patientin mit der Neuigkeit einer Spontanfraktur des 12. Brustwirbels mit der Diagnose massive Osteoporose. Diese wird nun nach weiteren 


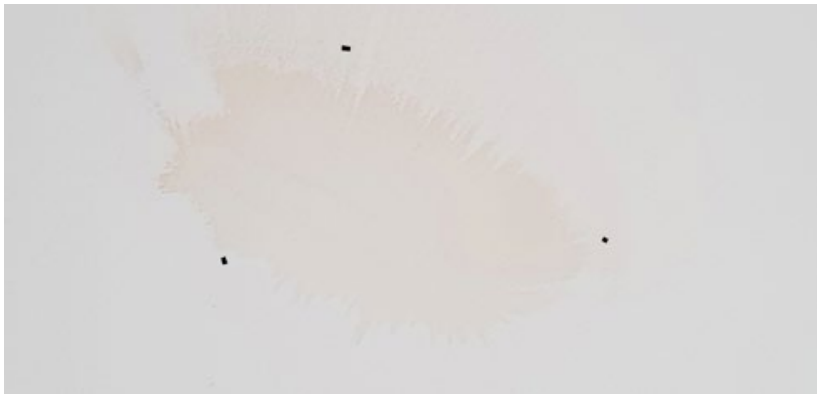

Abb. 1: Fleck an der Zimmerdecke: positiver RAC auf Aflatoxin

Abklärungen mit der Depotspritze Aclasta (Biphosphonat) Anfang Februar 2021 behandelt. 3 Wochen nach der Injektion kommt die Patientin wieder zu mir zur Kontrolle und klagt über erneut schlechten Schlaf, Müdigkeit, Appetitlosigkeit sowie erneut vermehrt Blasenbrennen, nachdem dieses Symptom vor der Injektion fast verschwunden war. Die aurikulomed. Untersuchung ergibt: Inversion links durch Störherd an linker Niere.

Da die Injektion gegen Osteoporose nicht sehr lange zurückliegt, liegt die Vermutung nahe, dass das infundierte Medikament Aclasta eine neue Störung verursacht hat. Der Störherd linke Niere verschwindet bei WegnahmeAuflage von Aclasta (als chemische Formel). Dies erklärt auch, warum die vorherige Besserung der Blasensymptomatik durch die gelungene Ausleitung von Aflatoxin sich wieder verschlechtert hat. Passender Symptompunkt ist nun wieder die Blase. Der TUP ist diesmal Punkt Le 13 (Stoffwechselfunktion).Wir finden nun zur Ausleitung der Störinformation, verursacht durch das infundierte Medikament Aclasta, Rosmarinus von Fa. Ceres, das zur Stoffwechselunterstützung zusätzlich verordnet wird.

Eine erneute Konsultation im April 2021: Blasenbeschwerden sind immer noch vorhanden (Aclasta-Einfluss) und meine Routine-Testung auf Coronavirus ergibt einen positiven RAC über Leber und Milz. Die Patientin ist derzeit auch müde und erschöpft, hatte aber sonst keine spezifischen Corona-Symptome verspürt. Ein schulmedizinischer Corona-Test fiel negativ aus, Infektion lag wohl länger zurück.

Positiv ist, dass in der Zwischenzeit Aflatoxin an der Patientin nicht mehr nachweisbar ist. Die neue Stoffwech-

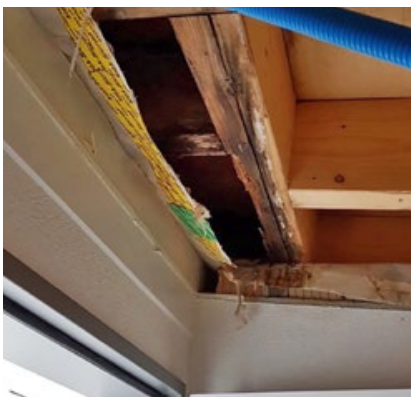

Abb. 2: Schaden im Gebälk

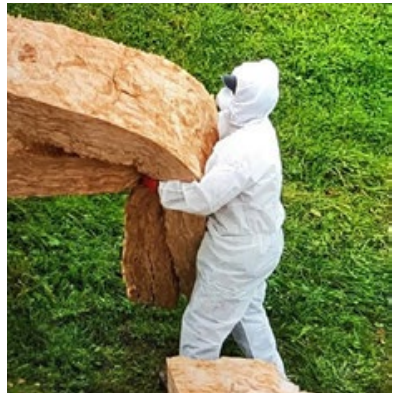

Abb. 3: Entfernung des Isolationsmaterials selbelastung ist jetzt verursacht durch COVID-19-Toxine, sichtbar über Störherd Leber (Resonanz mit der Information Corona-Spikeprotein/Potenz M 100), gekoppelt mit Vit. A, Symptompunkt ist Punkt Gb 43, TUP zeigt sich am Valiumpunkt, Psyche-Pkt: Ma 36. Die Patientin setzt ihre Therapie mit Desmodium fort, da letzteres bei dessen peripheren Auflage die Covid-Information löscht.

Dieser Fall illustriert, dass die klinischen Symptome isoliert $\mathrm{zu}$ betrachten und $\mathrm{zu}$ behandeln keine globale Lösung gebracht hätte. Das Umfeld, vorab die Wohnsituation, musste mit einbezogen und auch evaluiert werden. Ansonsten wären wir bei einer symptomatischen Behandlung ohne ursächliche Lösungen geblieben. Der zweite Störfaktor, welcher medikamentöser Natur ist und sich vor Abschluss der extern toxischen Belastung manifestiert, zeigt uns, dass der immer wieder erwähnte Grundsatz von Bahr - keine vorgefasste Meinung, sondern systematisches Vorgehen nach der aurikulomedizinischen Klassik - uns vor Fehlinterpretationen schützen kann. Hinter einer Störung können sich andere Störungen verbergen.

EinVorteil der Aurikulomedizin besteht darin, von vielfältigen Resonanztests Gebrauch zu machen, um erfolgreich auch schädliche Umfeldeinflüsse in Betracht ziehen zu können. In meinem Basis-Untersuchungs-Set für Störherde finden sich folgende Ampullen als Informationsträger:Virus, Bakterien, Parasiten, Würmer, Pilze, MullaMulla nach Heck (DE) für Umwelttoxine; letztere werden mit einem Spezialtestsatz evaluiert, können nicht selten auf Unverträglichkeiten im Bereich von Kosmetika und Haushaltchemikalien hinweisen, speziell auch bei Karzinompatienten.

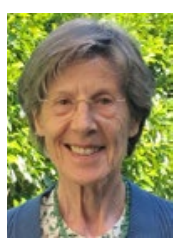

Dr. med. FA Gyn. Marianne Eggenberger

Aurikulomedizin ASA

Kornmarktgasse 4

CH-6004 Luzern

m-v.eggenberger@bluewin.ch 